\title{
ON FINITELY GENERATED MODULES OVER NOETHERIAN RINGS
}

\author{
BY \\ J. P. JANS(1)
}

0. Introduction. If $A$ is a module over a ring $R$, the module $\operatorname{Hom}_{R}(A, R)=A^{*}$ is usually called the dual of $A$. The elements of $A$ can be considered as homomorphisms from $A^{*}$ to $R$ so that there is a natural map

$$
A \rightarrow A^{* *}
$$

of $A$ into its second dual. Following the terminology introduced by Bass [2] we shall say that $A$ is torsionless if $\sigma$ is a monomorphism, reflexive if $\sigma$ is an isomorphism. We shall also refer to $\operatorname{Im} \sigma$ as the torsionless factor of $A$. It is easy to see that this is also $A / A_{0}$ where $A_{0}$ is the intersection of kernels of the elements of $A^{*}$.

In this paper we plan to continue the study initiated in [5] relating some of the concepts mentioned above with the functor $\operatorname{Ext}_{R}^{n}(A, R)$. Since it will only appear in this form in the present paper we shall henceforth adopt the notation $E^{n}(A)=\operatorname{Ext}_{R}^{n}(A, R)$. Also, in this paper we shall make the standing assumptions that the ring is both left and right Noetherian and all modules under consideration are finitely generated. The reason behind the latter assumption is to insure that projective modules are reflexive and that the duals of projective modules are projective; see [2].

In $\$ 1$ we relate double dual embeddings with the torsionless factors of modules $A$ such that $E^{1}(A)=0$. The proof of this result arose out of Theorem 1.4 of [5] in which it is shown that the first dual $A^{*}$ is a direct summand of the "third" dual $A^{* * *}$. In $\$ 2$ we introduce the concept of $D$-class $n$ and show that the dual of a module of $D$-class $n$ appears as the $n$th kernel in a projective resolution. It is then clear that properties of the ring which are phased in terms of the sort of kernels appearing in projective resolutions can also be described in terms of modules of $D$-class $n$ (and their duals).

In $\S 3$, we show that, modulo a special condition, a module $T_{n}$ is of $D$-class $n$ if and only if $E^{i}\left(T_{n}\right)=0$ for $1 \leqq i \leqq n-1$.

Under Applications, $\$ 4$, we relate properties of modules of $D$-class $n$ to the global dimension, left finitistic dimension and left injective dimension of the ring.

Received by the editors April 16, 1961 and, in revised form, January 8, 1962.

(1) Research supported in part by the United States Air Force Office of Scientific Research and in part by National Science Foundation contract NSF-G 11098. 
Most of these applications take the form of generalizations of theorems of [2; 5]. §5, Odds and ends, consists of some results which are easily proved by the methods of the paper.

1. $W$-modules and double dual embeddings. The following definitions will facilitate our exposition:

Definition. $A$ will be called a $W$-module if $E^{1}(A)=0$.

Definition. A monomorphism $X^{* *} \stackrel{\rho^{*}}{\rightarrow} F^{*}$ will be called a double dual embedding (D.D.E.) if it is the dual of an epimorphism $F \stackrel{\rho}{\rightarrow} X^{*}, X^{*}$ a dual.

The following theorem gives the the relation between double dual embeddings and the torsion factors of $W$-modules.

THEOREM 1.1. If $Q^{* *} \stackrel{\rho^{*}}{\rightarrow} F^{*}$ is a D.D.E. with $F$ projective then $F^{*} / \operatorname{Im} \rho^{*}$ is the torsionless factor of a W-module. Conversely, if $T$ is the torsionless factor of a W-module then there exists a projective module $F$ such that $0 \rightarrow X \stackrel{j}{\rightarrow} F \rightarrow T$ $\rightarrow 0$ is exact and $X \stackrel{j}{\rightarrow} F$ is a D.D.E.

Proof. Consider the exact sequence

$$
0 \rightarrow T^{*} \rightarrow F \stackrel{\rho}{\rightarrow} Q^{*} \rightarrow 0
$$

where

$$
0 \rightarrow Q^{* *} \stackrel{\rho^{*}}{\rightarrow} F^{*} \rightarrow T \rightarrow 0
$$

is also exact. $T$ is defined to be $F^{*} / \operatorname{Im} \rho^{*}$ and it turns out that $\operatorname{Ker} \rho=T^{*}$; this is really the argument in Bass' paper [2]. Choose in $Q^{* *}$ a submodule $Q$ such that $Q \stackrel{\sigma}{\rightarrow} Q^{* *}$ is the natural embedding. This gives rise to the following commutative diagram with exact rows and columns:

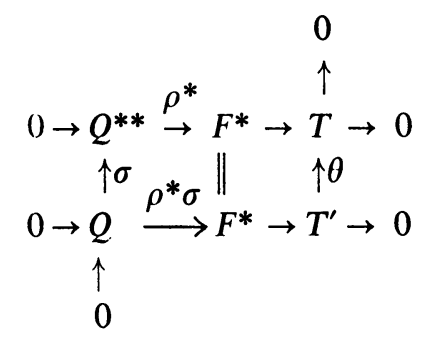

where $T^{\prime}$ is $F / \operatorname{Im}\left(\rho^{*} \sigma\right)$ and $\theta$ is the natural map of $F / \operatorname{Im}\left(\rho^{*} \sigma\right)$ onto $F / \operatorname{Im} \rho^{*}=T$.

Now dualize the entire diagram to obtain the following diagram:

$$
\begin{aligned}
& 0 \\
& \downarrow \\
0 \rightarrow & T^{*} \rightarrow F \stackrel{\rho^{* *}}{\rightarrow} Q^{* * *} \\
& \downarrow \theta^{*} \| \underset{\sigma^{*} \rho^{* *}}{\rightarrow}{ }^{*} \\
0 \rightarrow T^{*} \rightarrow & T^{1}\left(T^{\prime}\right) \rightarrow 0 .
\end{aligned}
$$


Note that the map $\sigma^{*}$ is an epimorphism and that $\operatorname{Im} \rho=\operatorname{Im} \rho^{* *}=Q^{*}$ embedded naturally in $Q^{* * *}$ [5]. It follows therefore that $\sigma^{*} \rho^{* *}$ is an epimorphism and consequently $T^{\prime}$ is a $W$-module. The "Five lemma" implies that $\theta^{*}$ is an isomorphism, and, in this way, $T$ can be considered as the torsionless factor of $T^{\prime}$ with $T^{\prime}$ a $W$-module.

To prove the converse statement, consider $T$ as the torsionless factor of $T^{\prime}$ with $E^{1}\left(T^{\prime}\right)=0$. Select $F$, projective mapping onto $T^{\prime}$ and construct the following commutative diagram with exact rows and columns:

where $\rho=\theta \mu$.

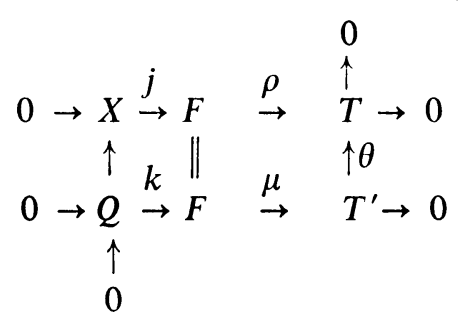

In the following we shall establish that $X \stackrel{j}{\rightarrow} F$ is a D.D.E. First form duals to obtain the diagram:

$$
\begin{aligned}
0 \rightarrow & T^{*} \stackrel{\rho^{*}}{\rightarrow} F^{*} \rightarrow F^{*} / \operatorname{Im} \rho^{*} \rightarrow 0 \\
& \downarrow \theta^{*} \mu^{*} \| \\
0 \rightarrow & T^{\prime *} \stackrel{\mu^{*}}{\rightarrow} F^{*} \rightarrow Q^{*} \rightarrow 0 .
\end{aligned}
$$

Exactness of the bottom row comes from the hypothesis $E^{1}\left(T^{\prime}\right)=0$. Also $\theta^{*}$ is an isomorphism by hypothesis. Since $\operatorname{Im} \mu^{*}=\operatorname{Im} \rho^{*}$ we have the following isomorphisms, $F^{*} / \operatorname{Im} \rho^{*}=F^{*} / \operatorname{Im} \mu^{*}=Q^{*}$. Thus we see that $F^{*} / \operatorname{Im} \rho^{*}$ is a dual $\left(Q^{*}\right)$ and it follows from [2] that the dual of the epimorphism $F^{*} \rightarrow F^{*} / \operatorname{Im} \rho^{*}$ is the monomorphism $X \stackrel{j}{\rightarrow} F$. The latter is therefore a D.D.E.

The above proof yields several corollaries.

Corollary 1.2. If $Q^{* *} \stackrel{\mu^{*}}{\rightarrow} F^{*}$ is a D.D.E. with $F$ projective and $Q^{* *}$ reflexive then $F^{*} / \operatorname{Im} \mu^{*}$ is a torsionless $W$-module. Conversely, if $T$ is a torsionless $W$ module, $0 \rightarrow X \stackrel{j}{\rightarrow} F \rightarrow T \rightarrow 0$ exact with $F$ projective then $X \stackrel{j}{\rightarrow} F$ is a D.D.E.

Proof. Recall that in the proof of the theorem we selected a module $Q$ in $Q^{* *}$ so that $Q \rightarrow Q^{* *}$ was the natural embedding of $Q$ in its double dual. In the corollary we can let $Q=Q^{* *}$ and the resulting diagram has only one row instead of two. In this case the $W$-module $T^{\prime}$ coincides with its torsionless factor $T$ and the first part of the corollary follows.

The converse part of the corollary follows from the proof of the corresponding part of the theorem using the fact that $T$ is its own torsionless factor.

In [2], Bass showed how to inject any torsionless module into a projective module by using the following construction. Let $T$ be torsionless and let $T^{*}$ be its dual. Find a projective $F$ mapping onto $T^{*}, F \stackrel{\mu}{\rightarrow} T^{*}$ and dualize to obtain 
the D.D.E. $T^{* *} \stackrel{\mu^{*}}{\rightarrow} F^{*}$. Since $T$ is torsionless the natural mapping of $T$ into $T^{* *}$ is a monomorphism which together with $\mu^{*}$ gives an embedding of $T$ into $F^{*}$. We shall call such an embedding of a torsionless module a standard embedding. Notice that the above construction makes up part of the diagrams used in the proof of Theorem 1.1 above. By arguing from these diagrams we get another corollary.

COROLLARY 1.3. The exact sequence $0 \rightarrow T \stackrel{\dot{j}}{\rightarrow} F$ is a standard embedding of the torsionless module $T$ in the projective $F$ if and only if $F / \operatorname{Im} j$ is $a W$-module.

We remark at this point that not all embeddings of torsionless modules in projectives are standard embeddings. For example, if $P \stackrel{\dot{m}}{\rightarrow} F$ is an embedding of a projective $P$ in the projective $F$ the embedding will be standard if and only if the embedding splits. If the embedding does not split then $F / \operatorname{Im} j$ has dimension one and modules of dimension one are never $W$-modules [4, p. 123]. Of course if the embedding splits it will be standard.

2. D-classes. In his paper Bass proved the following extremely useful theorem connecting arbitrary finitely generated modules and duals.

SHIFTING THEOREM. Let $A$ be finitely generated, $B$ torsionless $C^{*} a$ dual. If any one of the modules $A, B$ or $C^{*}$ is given the other two exist and are connected by the exact sequence

$$
0 \rightarrow C^{*} \rightarrow F \rightarrow B \rightarrow 0 \rightarrow B \rightarrow F^{\prime} \rightarrow A \rightarrow 0
$$

where $F$ and $F^{\prime}$ are projective.

We would like to extend this theorem (and some of its numerous corollaries) by shifting through projective modules even farther back. That is, we wish to examine the structure of the modules $D$ such that $0 \rightarrow D^{*} \rightarrow F^{\prime \prime} \rightarrow C^{*} \rightarrow 0$ is exact with $F^{\prime \prime}$ projective. While we are at it we might just as well shift back $n$ steps. The investigation will be facilitated by the following definition.

Definition. We shall say that the torsionless module $T_{n}$ is of $D$-class $n$ if it can be fitted into an exact diagram of the form

$$
\begin{aligned}
& 0 \quad \rightarrow \quad T_{n-1}^{* *} \rightarrow F_{n-1} \rightarrow T_{n} \rightarrow 0 \\
& \ldots \rightarrow F_{n-2} \rightarrow T_{n-1}^{\uparrow} \rightarrow 0 \\
& 0 \rightarrow T_{2}^{* *} \rightarrow \ldots \\
& 0 \rightarrow T_{1}^{* *} \rightarrow F_{1} \rightarrow T_{2} \rightarrow 0
\end{aligned}
$$

where each $F_{i}$ is projective, the horizontal monomorphisms are D.D.E.'s and the 
vertical maps are all natural embeddings of the $T_{i}$ in their second duals $T_{i}^{* *}$. We shall say that any torsionless module is of $D$-class 1 .

The following theorem establishes a connection between (left) modules of $D$-class $n$ and the kernels in a projective resolution of a (right) module.

THEOREM 2.1. If there exists a collection of exact sequences

$$
0 \rightarrow T_{i+1}^{*} \rightarrow F_{i} \rightarrow T_{i}^{*} \rightarrow 0, \quad 1 \leqq i \leqq n-1
$$

with each $F_{i}$ projective then $T_{n}$ can be selected of D-class $n$. Conversely, if $T_{n}$ is a module of D-class $n$ then $T_{n}^{*}$ can be embedded in a collection of the form $\left(1^{*}\right)$.

Proof. As at the beginning of the proof of Theorem 1.1, the sequence $0 \rightarrow T_{i+1}^{*} \rightarrow F_{i} \rightarrow T_{i}^{*} \rightarrow 0$ induces the sequence $0 \rightarrow T_{i}^{* *} \rightarrow F_{i}^{*} \rightarrow T_{i+1} \rightarrow 0$ where the monomorphism is clearly a D.D.E. Now we can obtain a diagram of the form (1) by stringing these sequences together with the natural vertical maps $T_{i} \rightarrow T_{i}^{* *}$. Thus $T_{n}$ can be chosen to be of $D$-class $n$.

Conversely, dualizing a sequence of the form $0 \rightarrow T_{i}^{* *} \rightarrow F_{i} \rightarrow T_{i+1} \rightarrow 0$ gives rise to a sequence of the form $0 \rightarrow T_{i+1}^{*} \rightarrow F_{i}^{*} \rightarrow T_{i}^{* * *}$. But since we are assuming that the injection in the first sequence is a D.D.E., the map from $F_{i}^{*}$ to $T_{i}^{* * *}$ in the second sequence is the second dual of an epimorphism of $F_{i}^{*}$ onto $T_{i}^{*}$. Because projectives are reflexive that second dual will coincide with the original map, and we obtain the sequence $0 \rightarrow T_{i+1}^{*} \rightarrow F_{i}^{*} \rightarrow T_{i}^{*} \rightarrow 0$. That is, if $T_{n}$ is a module of $D$-class $n, T_{n}^{*}$ can be embedded in a diagram of the form (1*).

It should be noted that every module involved in the definition of $D$-classes and in the proof of Theorem 2.1 is torsionless. The whole thing can be rephrased in nontorsionless terms and a corresponding theorem can be proved. For the sake of completeness, we indicate briefly how this can be done.

Definition. $T_{n}^{\prime}$ will be of $D^{\prime}$-class $n$ if it can be fitted into an exact diagram of the form

$$
\begin{aligned}
& 0 \quad \rightarrow \quad T_{n-1} \rightarrow F_{n-1} \rightarrow T_{n}^{\prime} \rightarrow 0 \\
& \uparrow \\
& F_{n-2} \rightarrow T_{n-1}^{\prime} \rightarrow 0 \\
& 0 \rightarrow T_{2} \rightarrow \\
& 0 \rightarrow T_{1} \rightarrow F_{1} \rightarrow T_{2}^{\prime} \rightarrow 0
\end{aligned}
$$

where each $T_{i}^{\prime}$ is a $W$-module for $2 \leqq i \leqq n$ and all the vertical maps are the mappings of the $T_{i}^{\prime}$ on the torsionless factors $T_{i}$. We shall allow any module to be of $D^{\prime}$-class 1 .

The theorem that would go with the above definition would read like Theorem 2.1 with $D^{\prime}$ and $T_{n}^{\prime}$ replacing $D$ and $T_{n}$. That one can jump back and forth 
between the diagrams (1) and ( $\left.1^{\prime}\right)$ is the content of Theorem 1.1. Moreover that theorem also shows that a module is of $D$-class $n$ if and only if it is the torsionless factor of a module of $D^{\prime}$-class $n$. Note that the modules of $D^{\prime}$-class 2 are the $W$-modules.

3. Ext of modules of $D$-class $n$. If one can find a torsionless module $T_{n}$ with the property that $E^{i}\left(T_{n}\right)=0$ for $1 \leqq i \leqq n-1$ then it is easy to see that the module is of $D$ class $n$. For if

$$
0 \rightarrow T_{1} \rightarrow F_{1} \rightarrow F_{2} \rightarrow \ldots F_{n-1} \rightarrow T_{n} \rightarrow 0
$$

is part of a projective resolution for $T_{n}$ with $F_{i}$ projective then a repeated application of Corollary 1.2 yields the diagram

$$
\begin{aligned}
& 0 \quad \rightarrow \quad T_{n-1}^{* *} \rightarrow F_{n-1} \rightarrow T_{n} \rightarrow 0 \\
& F_{n-2} \rightarrow T_{n-1}^{* *} \rightarrow 0 \\
& 0 \rightarrow T_{1}^{* *} \rightarrow F_{1} \rightarrow T_{2}^{* *} \rightarrow 0
\end{aligned}
$$

where the $T_{i}^{* *}$ are the appropriate kernels.

In this section we investigate a condition which will insure that all modules $T_{n}$ of $D$-class $n$ have the property that $E^{i}\left(T_{n}\right)=0$ for $1 \leqq i \leqq n-1$. That is we shall try to collapse the diagram (1) what is really an exact sequence (2).

At this point the exposition is facilitated by the concept of grade, defined by Rees [6] for commutative Noetherian rings.

Definition. The module $M$ has grade $r$ if $E^{i}(M)=0$ for $i \leqq r-1$ and $E^{r}(M)$ $\neq 0$. The module $S$ has reduced grade $r$ if $E^{i}(S)=0$ for $1 \leqq i \leqq r-1$ and $E^{r}(S) \neq 0$.

Note that torsionless modules always have grade 0 (since $E^{0}(T) \neq 0$ ) but the reduced grade of a torsionless module may be large. In fact our goal in this section is to prove (under certain conditions) that if $T_{n}$ is of $D$-class $n$ then the reduced grade of $T_{n}$ is greater than or equal to $n-1$.

THEOREM 3.1. If $R$ has the property that for all $r$ and for all finitely generated right modules $M$ the grade of $E^{r}(M)$ is greater than $r-1$ then every left module of D-class $n$ has reduced grade greater than $n-1$.

Proof. Since for $n=1$ there is nothing to prove, we begin an induction at $n=2$. In this case the hypothesis implies that $E^{0}\left(E^{2}(M)\right)=0$ for all finitely generated right modules $M$. Now by Corollary 1.5 of [5] we know that the duals of all left modules are reflexive. But then $T^{* *}$ is reflexive for each left module $T$ and we can conclude from Corollary 1.2 that every left module $T$ of $D$-class $n(n \geqq 2)$ is a $W$-module. In particular if $T_{2}$ is of $D$-class 2 th:n $E^{1}\left(T_{2}\right)=0$ and the reduced grade of $T_{2}$ is greater than 1 . 
Assume now that the theorem has been established for integers less than $n$ and that $n$ is greater than 2. Since $T_{n-1}$ in the diagram (1) is of $D$-class $n-1$ we have $E^{j}\left(T_{n-1}\right)=0$ for $1 \leqq j \leqq n-2$. To finish the proof we shall show that $E_{j}\left(T_{n-1}\right)$ $=E^{j}\left(T_{n-1}^{* *}\right)$ for $1 \leqq j \leqq n-2$. This will be sufficient for we see that the equation $E^{j}\left(T_{n-1}^{* *}\right)=E^{j+1}\left(T_{n}\right)$ holds for all $j \geqq 1$. Also we know that $E^{1}\left(T_{n}\right)=0$ from the first part of the proof.

If we examine the diagrams $(1)$ and $\left(1^{*}\right)$ and apply the duality theorem of [5] we obtain the short exact sequence

$$
0 \rightarrow T_{n-1} \rightarrow T_{n-1}^{* *} \rightarrow E^{1}\left(T_{n-2}^{*}\right) \rightarrow 0 .
$$

Also, from the sequence $\left(1^{*}\right)$ we get the isomorphism $E^{1}\left(T_{n-2}^{*}\right)=E^{n-2}\left(T_{1}^{*}\right)$. By an application of Bass' Shifting Theorem we can raise the superscript by two to obtain $E^{n}(M)=E^{n-2}\left(T_{1}^{*}\right)$ for a suitable right module $M$. If we put this into the sequence (3) we have the short exact sequence

$$
0 \rightarrow T_{n-1} \rightarrow T_{n-1}^{* *} \rightarrow E^{n}(M) \rightarrow 0 .
$$

Now apply $E^{j}$ to this sequence and use the hypothesis that $E^{j}\left(E^{n}(M)\right)=0$ for $0 \leqq j \leqq n-1$. From the exact sequence of homology we obtain the desired isomorphisms $E^{j}\left(T_{n-1}\right)=E^{j}\left(T_{n-1}^{* *}\right)$ holding for $1 \leqq j \leqq n-2$. This completes the proof of the theorem. We remark that the proof of the theorem did not use the full force of the hypothesis "all $r$ " but uses instead the hypothesis "all $r$ up to and including $n . "$

The rather strange hypothesis "grade $E^{r}(M)$ greater than $r-1$ " in the preceding theorem brings up the question of which rings have this condition. H. Bass has constructed a proof of the fact that for a commutative Noetherian ring $R$ this condition is equivalent to the condition that $R_{p}$ has finite injective dimension over itself for every prime $p$ where $R_{p}$ is the localization of $R$ at $p$. The proof of this can be based on the results of [3]. We know of no analogous theorem for noncommutative rings.

4. Applications. In this section we relate modules of $D$-class $n$ to various invariants of the ring. Among these invariants are the global dimension, left (and right) finitistic dimension and the left (and right) injective dimension of the ring as a module over itself. Recall that the global dimension of $R, \mathrm{gl} \cdot \operatorname{dim} \cdot R$, is defined to be the supremum of the projective dimensions of all the $R$-modules Auslander showed [1], that for the rings we consider this can be computed by taking the supremum of the dimensions of the cyclic left $R$-modules. The left finitistic dimension of $R, \operatorname{lfP}(R)$, is the supremum of the projective dimensions of the finitely generated left modules of finite projective dimension. See Bass' paper [2] for a number of relations between these and other dimensions.

The following theorem connects a property of modules of $D$-class $n$ with the global dimension of the ring. 
THEOREM 4.1. For the rings $R$ under consideration the following are equivalent for integers $n \geqq 1$ :

(a) $\operatorname{gl}$.dim. $(R) \leqq n+1$.

(b) Duals of modules of D-class $n$ are projective.

Proof. Combining Bass' Shifting Theorem with Theorem 2.1 we see that if $T_{n}^{*}$ is the dual of a right module of $D$-class $n$ then $T_{n}^{*}$ appears as the kernel in the $n$th projective module of a projective resolution of some left $R$-module $A$. And, conversely, given a projective resolution of some left $R$-module $A$ the $n$th kernel is the dual of a right module of $D$-class $n$. Thus (a) and (b) are equivalent.

We remark that we mean by the $n$th module in a projective resolution, the one with subscript $n$. The subscripts start at zero. This is the usual notation, but it is a poor way to count.

In the above argument, we did not need to distinguish between left and right because of Auslander's result mentioned above. In the next theorem, we do have to make such a distinction since the left and right finitistic dimensions need not be the same. The following theorem can be considered as a generalization of Theorem 5.3 of [2].

THEOREM. 4.2. For the rings under consideration the following are equivalent for $n \geqq 1$ :

(a) lf $P D(R) \leqq n$.

(b) The only right modules of D-class $n$ with projective duals are the projectives.

Proof. For $n=1$ this is exactly Bass' Theorem. Assume condition (a) and let $T_{n}$ be of $D$-class $n$ with $T_{n}^{*}$ projective. By the proof of Theorem $4.1, T_{n}^{*}$ is the kernel in the $n$th projective module of a projective resolution of some left module $A$, and $A$ is therefore of finite projective dimension. By the assumption (a) we see that the sequence $0 \rightarrow T_{n}^{*} \rightarrow F_{n-1} \rightarrow T_{n-1}^{*} \rightarrow 0$ is exact and splits. But then the dual sequence $0 \rightarrow T_{n-1}^{* *} \rightarrow F_{n-1}^{*} \rightarrow T_{n} \rightarrow 0$ used in showing $T_{n}$ to be of $D$-class $n$ also splits, and $T_{n}$ is projective.

Conversely, assume condition (b) and let $A$ be a left module of projective dimension less than or equal to $n+1$ for $n \leqq 2$. We will show that its dimension is actually less than $n+1$. If $F$ is the $n$th projective in a projective resolution of $A$, we have the sequence $0 \rightarrow T_{n}^{*} \rightarrow F \rightarrow T_{n-1}^{*}$ where $T_{n}$ is of $D$-class and $T_{n}^{*}$ is projective. By (b) we conclude that $T_{n}$ is projective and the sequence $0 \rightarrow T_{n-1}^{* *} \rightarrow F^{*} \rightarrow T_{n}$ $\rightarrow 0$ used in exhibiting the $D$-class of $T$ splits. Thus we see that $T_{n-1}^{* *}$ is projective. But $T_{n-1}^{*}$ is a direct summand of $T_{n-1}^{* * *}$ so both of these are projective. That is, the projective dimensions of $A$ is less than $n+1$. This concludes the proof of the theorem.

In [5] we showed that the difference between a torsionless module and its second dual is a module of the form $E^{1}(B)$. We were able to use this to connect 
the vanishing of $E^{1}(B)$ and $\left[E^{1}(B)\right]^{*}$ with reflexiveness. The following theorem can be thought of as an extension of Corollary 1.3 of [5].

THEOREM 4.3. For the rings $R$ under consideration the following are equivalent for $n \geqq 1$ :

(a) The left modules of $D$-class $n$ are reflexive.

(b) $E^{n}(B)=0$ for all torsionless right modules $B$.

(c) $E^{n+1}(C)=0$ for all right modules $C$.

(d) The right injective dimension of the ring is less than or equal to $n$.

Proof. For $n=1$ this is exactly Corollary 1.3 of [5]. Also the equivalence of (b), (c), and (d) for all $n \geqq 1$ follows from Bass' Shifting Theorem. It is therefore sufficient to show the equivalence of (a) and (b) for $n \geqq 2$.

If $T_{n}$ is of $D$-class $n$ then we are assured of the two sequences

$$
\begin{aligned}
& 0 \rightarrow T_{n-1}^{* *} \rightarrow F^{*} \rightarrow T_{n} \rightarrow 0, \\
& 0 \rightarrow T_{n}^{*} \rightarrow F \rightarrow T_{n-1}^{*} \rightarrow 0 .
\end{aligned}
$$

Under these circumstances the conclusion of Theorem 1.1 of [5] holds and we obtain the additional exact sequence

$$
0 \rightarrow T_{n} \rightarrow T_{n}^{* *} \rightarrow E^{1}\left(T_{n-1}^{*}\right) \rightarrow 0 .
$$

Since $T_{n}$ was of $D$-class $n, T_{n-1}^{*}$ can be thought of as a kernel in a projective resolution of a torsionless module $B$. By using the exact sequence of homology on $E^{i}(\cdot)$ we see that $E^{1}\left(T_{n-1}^{*}\right)=E^{n}(B)$. Thus we arrive at the exact sequence

$$
0 \rightarrow T_{n} \rightarrow T_{n}^{* *} \rightarrow E^{n}(B) \rightarrow 0 \text {. }
$$

The above construction can be reversed in the sense that we could have started with the torsionless right module $B$ and worked backwards to get a left module of $D$-class $n$. It is clear now that the sequence $\left(^{*}\right)$ gives the equivalence of (a) and (b).

We know from [5] that we can hang stars on the sequence $\left(^{*}\right)$ to get the following exact split sequence,

$$
0 \rightarrow\left[E^{n}(B)\right]^{*} \rightarrow T_{n}^{* * *} \rightarrow T_{n}^{*} \rightarrow 0 .
$$

From this sequence we get immediately the following corollary.

COROLLARY 4.4. For the rings under consideration the following are equivalent for $n \geqq 1$ :

(a) $\left[E_{n}(B)\right]^{*}=0$ for all torsionless right modules $B$.

(b) $T^{*}$ is reflexive for all left modules $T_{n}$ of D-class $n$.

5. Odds and ends. We include the following because the methods of proof appear to be related to the preceding results. 
THEOREM 5.1. For the rings under consideration the following conditions are equivalent:

(a) All left $W$-modules are torsionless.

(b) All right torsionless modules are $W$-modules.

(c) All torsionless left modules are reflexive.

(d) The right injective dimension of the ring is less than or equal to one.

Proof. We showed the equivalence of (b), (c) and (d) in Corollary 1.3 of [5]. In the following we shall establish the equivalence of (a) and (b).

Let $A$ be a torsionless right module. From Theorem 1.1 of [5] there is a torsionless left module $B$ such that $0 \rightarrow B \rightarrow B^{* *} \rightarrow E^{1}(A) \rightarrow 0$ is exact. By the proof of Theorem 1.1 we can embed this sequence in an exact diagram

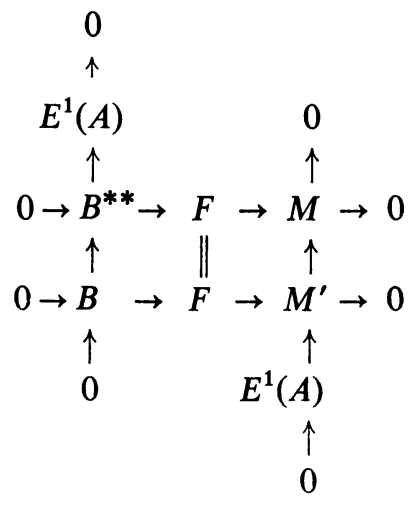

where $E^{1}\left(M^{\prime}\right)=0, M$ is the torsionless factor of $M^{\prime}$, and $F$ is projective.

If condition (a) holds, we have $M=M^{\prime}$ and $B=B^{* *}$ so that $E^{1}(A)=0$. Then (a) implies (b). By Theorem 1.1 we could have started with the left $W$-module $M^{\prime}$ and formed the above diagram with $A$ torsionless right module. Assuming condition (b) $E^{1}(A)=0$ and it follows that $M^{\prime}$ is its own torsionless factor. This completes the proof of the theorem.

The following may be well known, but we include it for laughs. It seems to be related to Theorem 4.1 .

THEOREM 5.2. For the rings $R$ under consideration the following are equivalent:

(a) All $W \cdot$ modules are projective.

(b) $\operatorname{gldim} .(R) \leqq 1$.

Proof. If (b) holds then $R$ has only modules of dimensions zero and one. If $A$ has dimension one then by $\left[4\right.$, p. 123] $E^{1}(A) \neq 0$ and (a) follows:

Now assume (a). Let $B$ be a torsionless module, we shall show that $B$ is projective. By Corollary 1.3 , we can embed $B$ in a projective so that the factor is a $W$ module. But then the embedding splits and $B$ is therefore projective. 
In [5] we showed that if $B^{*}=0$ then $B=E^{1}(A)$ for a suitable $A$ of dimension one (or zero if $B$ is projective). Some of our above arguments show that we can find modules of the form $E^{1}(A)$ in another way.

THEOREM 5.3. If $M^{\prime}$ is a $W$-module then the kernel of the map of $M^{\prime}$ on its torsionless factor is $E^{1}(A)$ for some torsionless $A$. Conversely, for every torsionless $A$ there is a $W$-module $M^{\prime}$ such that $E^{1}(A)$ is the kernel of the map of $M^{\prime}$ on its torsionless factor.

Proof. The proof consists of examining the big diagram used in the proof of Theorem 5.1 and the fact that the diagram can be constructed starting either with the right module $A$ of with the left $W$-module $M^{\prime}$.

It should be noted that in the above theorem $M^{\prime}$ and $A$ are modules of the opposite hand.

\section{BIBLIOGRA PHY}

1. M. Auslander, On the dimension of modules and algebras. III. Global dimension, Nagoya Math. J. 9 (1955), 67-76.

2. H. Bass, Finitistic dimension and a homological generalization of semi-primary rings, Trans. Amer. Math. Soc. 95 (1960), 466-488.

3. - Injective dimension in Noetherian rings, Trans. Amer. Math. Soc. 102 (1962), 18-29.

4. H. Cartan and S. Eilenberg, Homological algebra, Princeton Univ. Press, Princeton, N. J., 1956.

5. J. P. Jans, Duality in Noetherian rings, Proc. Amer. Math. Soc. 12 (1961), 829-835.

6. D. Rees, The grade of an ideal or module, Proc. Cambridge Philos. Soc. 53 (1957), 28-42.

UNIVERSITY OF WASHINGTON,

SEATTLE, WASHINGTON 